Mycosphere 7 (2): 204-213 (2016) www.mycosphere.org ISSN 20777019

Article

Mycosphere

Doi 10.5943/mycosphere/7/2/9

Copyright (C) Guizhou Academy of Agricultural Sciences

\title{
Two new Seimatosporium species from Italy
}

\section{Wijayawardene $\mathrm{NN}^{12}$, Goonasekara $\mathrm{ID}^{2}$, Camporesi $\mathrm{E}^{3}$, Wang $\mathrm{Y}^{4}$ and $\mathrm{An} \mathrm{YL}^{1}$}

\author{
${ }^{1}$ The Key Laboratory of Karst Environment and Geohazard Prevention, Guizhou University, P.R. China \\ ${ }^{2}$ Center of Excellence in Fungal Research and School of Science, Mae Fah Luang University, Chiang Rai, 57100, \\ Thailand \\ ${ }^{3}$ A.M.B. Gruppo Micologico Forlivese "Antonio Cicognani", Via Roma 18, Forli, Italy; A.M.B. Circolo Micologico \\ "Giovanni Carini”, C.P. 314, Brescia, Italy; Società per gli Studi Naturalistici della Romagna, C.P. 144, \\ Bagnacavallo (RA), Italy \\ ${ }^{4}$ Department of Plant Pathology, College of Agriculture, Guizhou University, Guiyang, Guizhou 550025, P.R. China
}

Wijayawardene NN, Goonasekara ID, Camporesi E, Wang Y, An YL 2016 - Two new Seimatosporium species from Italy. Mycosphere 7(2), 204-213, Doi 10.5943/mycosphere/7/2/9

\begin{abstract}
Two taxa resembling Seimatosporium and Seiridium were collected from Italy. Mega blast results of ITS and LSU sequence data, showed that new collections are related to Seimatosporium. Parsimonious analyses based on LSU and ITS sequence data showed that new taxa reside in Seimatosporium sensu stricto. Based on morphological and molecular analyses, the new collections are introduced as new species and compared with taxa with similar morphological characters and host association.
\end{abstract}

Keywords - Coelomycetes - morphology - multi-gene - phylogeny

\section{Introduction}

The genus Seimatosporium Corda was introduced by Corda (1833) with S. rosae Corda as the type species. The genus is characterised by holoblastic to annellidic conidiogenous cells and cylindrical, fusiform or clavate or obovoid conidia, with brown median cells and (2-)3(-5)-septa (Sutton 1980, Nag Raj 1993, Barber et al. 2011, Tanaka et al. 2011, Senanayake et al. 2015, Norphanphoun et al. 2015, Wijayawardene et al. 2016). Conidia may entirely lack appendages, have only apical or basal appendages or have both apical and basal appendages (Norphanphoun et al. 2015, Wijayawardene et al. 2016). Recent phylogenetic studies showed that Seimatosporium resides in Discosiaceae, Amphisphaeriales (Senanayake et al. 2015, Norphanphoun et al. 2015, Wijayawardene et al. 2016). Tanaka et al. (2011) showed that Seimatosporium groups with Discostroma as a monotypic clade and this was confirmed by Senanayake et al. (2015), Norphanphoun et al. (2015) and Wijayawardene et al. (2016).

In this paper, we introduce two new species of Seimatosporium based on morpho-molecular analyses. Morphological characters and taxonomic keys in Sutton (1980), Nag Raj (1993) and recently published articles (Ariyawansa et al. 2015, Senanayake et al. 2015, Norphanphoun et al. 2015) were used to compare morphological characters. The phylogenetic analyses were carried out based on LSU and ITS sequence data. 


\section{Materials and Methods}

\section{Collection, isolation and morphological studies}

Decayed plant materials were collected from Italy, and placed in paper bags and/or Zip-lock bags. The samples were observed with a stereo microscope to detect fruit bodies. Sterilized needles were used to pick conidiomata and squash mounts were made to reveal the micro- morphological characters viz. conidiophores, conidiogenous cells, conidiogenesis and conidia (Sutton 1980). Vertical sections of conidioma were made using razor blades to examine the shape of conidioma and arrangement of conidiophores and conidiogenous cells. Morphological characters were examined under a compound microscope (Nikon Eclipse E600 DIC microscope and a Nikon DSU2 camera or a Nikon Eclipse 80i compound microscope fitted with a Canon 450D digital camera).

Isolation was carried out as detailed in Chomnunti et al. (2014) and germinating conidia were transferred aseptically to potato dextrose agar (PDA). Germinating conidia were transferred to PDA plates and incubated at $18{ }^{\circ} \mathrm{C}$ for further growth. Colony colour and other characters were assessed after 1 to 2 weeks. The holotype specimens are deposited in the Mae Fah Luang University Herbarium (MFLU), Chiang Rai, Thailand. Ex-type cultures are also deposited in Culture Collection at Mae Fah Luang University (MFLUCC) and Department of Plant Pathology, Agriculture College, Guizhou University, P.R. China (GUCC). Facesoffungi and Index Fungorum numbers are provided as explained in Jayasiri et al. (2015) and Index Fungorum (2016)

\section{DNA extraction, PCR amplification and sequencing}

Colonies generated from germinated single conidia were further grown on PDA for 14 days at $18{ }^{\circ} \mathrm{C}$. Fresh fungal mycelia were scraped from PDA using sterilized scalpels. A BIOMIGA Fungus Genomic DNA Extraction Kit (GD2416) was used to extract DNA from the scraped mycelia. The amplification of rDNA regions of the internal transcribed spacers (ITS) and large subunit (LSU) genes was carried out by using primers ITS5 and ITS4 and LROR and LR5 (Vilgalys and Hester 1990, White et al. 1990). Optimum conditions for amplification of ITS and LSU regions are as described in Alves et al. (2004). Amplified PCR fragments were checked on 1\% agarose electrophoresis gels stained with ethidium bromide. Purified PCR products (by minicolumns, purification resin and buffer according to the manufacturer's protocols Amersham product code: 27-9602-01) were sent to SinoGenoMax Co., Beijing, China for DNA sequencing. The nucleotide sequence data obtained are submitted to GenBank (Table 1).

\section{Phylogenetic analyses}

A megablast search was carried out to confirm the placement of the new strains in Amphisphaeriales and therefore phylogenetically related sequences were downloaded from GenBank (Table 1). However, only two strains were successful in single spore isolation, thus, only two strains were used in the phylogenetic analyses. Since both strains show a closer relationship with Seimatosporium and Discostroma, Ariyawansa et al. (2015), Senanayake et al. (2015) and Norphanphoun et al. (2015) were used to select strains for the phylogenetic analyses. Sequences for each gene region (LSU and ITS) were aligned using MAFFTv6 (Katoh et al. 2002, Katoh and Toh 2008), and online sequence alignment was edited under the default settings (mafft.cbrc.jp/alignment/server/). All absent genes were coded as missing data.

Combined LSU and ITS datasets was performed using maximum parsimony (MP) and Bayesian Posterior Probabilities (BYPP). Maximum-parsimony analyses were performed by PAUP v. 4.0b10 (Swofford 2002) using the heuristic search option with 1,000 random taxa addition and tree bisection and reconnection (TBR) as the branch swapping algorithm. All characters were unordered and of equal weight and gaps were treated as missing data. The Tree Length (TL), Consistency Indices (CI), Retention Indices (RI), Rescaled Consistency Indices (RC) and Homoplasy Index (HI) were calculated for each tree generated. 
Table 1 Strains used in this study

\begin{tabular}{|c|c|c|c|}
\hline \multirow[t]{2}{*}{ Taxon } & \multirow[t]{2}{*}{ Culture collection no. } & \multicolumn{2}{|c|}{ GenBank Accession no. } \\
\hline & & $L S U$ & ITS \\
\hline Adisciso yukushimense & MAFF 242774 & AB593721 & - \\
\hline Adisciso tricellulare & NBRC 32705 & AB593728 & - \\
\hline Discosia artocreas & NBRC 8975 & AB593705 & - \\
\hline Discosia pini & MAFF 410149 & AB593708 & AB594776 \\
\hline Discosia pseudoartocreas & CPC 21117 & KF777214 & - \\
\hline Discosia aff pleurochaeta & MAFF 242778 & AB593709 & AB594777 \\
\hline Discosia aff pleurochaeta & MAFF 242779 & AB593713 & AB594781 \\
\hline Discosia aff. brasiliensis & MAFF 237018 & AB593719 & AB594787 \\
\hline Discostroma botan & HHUF 4642 & DQ368629 & - \\
\hline Discostroma fuscellum & MFLUCC 14-0052 & KT005514 & KT005515 \\
\hline Discostroma fuscellum & NBRC 32680 & AB593739 & AB594806 \\
\hline Discostroma stoneae & NBRC 32690 & AB593729 & AB594797 \\
\hline Discostroma tostum & NBRC 32626 & AB593727 & AB594795 \\
\hline Pseudopestalotiopsis theae & MFLUCC 12-0055 & KM116282 & JQ683727 \\
\hline Sarcostroma bisetulatum & CBS 122695 & - & EU552155 \\
\hline Sarcostroma lomatiae & CBS 118144 & DQ278926 & DQ278921 \\
\hline Sarcostroma restionis & CBS 118153 & DQ278925 & DQ278923 \\
\hline Sarcostroma restionis & CBS 118154 & DQ278924 & DQ278922 \\
\hline Seimatosporium azaleae & MAFF 237478 & AB593730 & AB594798 \\
\hline Seimatosporium biseptatum & CPC 13584 & JN871208 & JN871199 \\
\hline Seimatosporium botan & Н 4619 & AB593731 & AB594799 \\
\hline Seimatosporium botan & HMUC 316PD & AB594799 & - \\
\hline Seimatosporium cornii & MFLUCC 14-0467 & KR559739 & KT162918 \\
\hline Seimatosporium discosioides & H 4621 & AB593732 & AB594800 \\
\hline Seimatosporium elegans & NBRC 32674 & AB593733 & AB594801 \\
\hline Seimatosporium eucalypti & CPC 156 / CBS 115131 & JN871209 & JN871200 \\
\hline Seimatosporium eucalypti & CPC 157 / CBS 110733 & JN871210 & JN871201 \\
\hline Seimatosporium eucalypti & CPC 158 / CBS 110734 & JN871211 & - \\
\hline Seimatosporium eucalypti & CPC 159 / CBS 114876 & JN871212 & JN871202 \\
\hline Seimatosporium falcatum & CPC 12992 & - & JN871203 \\
\hline Seimatosporium falcatum & CPC 13578 & JN871213 & JN871204 \\
\hline Seimatosporium falcatum & CPC 13580 & JN871214 & JN871205 \\
\hline Seimatosporium ficeae & MFLUCC 15-0519 & KR920686 & - \\
\hline Seimatosporium foliicola & NBRC 32676 & AB593734 & AB594802 \\
\hline Seimatosporium glandigenum & NBRC 32677 & AB593735 & AB594803 \\
\hline Seimatosporium grevilleae & ICMP 10981 & AF382372 & AF405304 \\
\hline Seimatosporium hakeae & NBRC 32678 & AB593736 & AB594804 \\
\hline Seimatosporium hypericinum & NBRC 32647 & AB593737 & AB594805 \\
\hline Seimatosporium kriegerianum & NBRC 32679 & AB593738 & - \\
\hline Seimatosporium leptospermi & ICMP 11845 & AF382373 & - \\
\hline Seimatosporium lichenicola & MFLUCC 14-0623 & KT198725 & KT198724 \\
\hline Seimatosporium mariae & NBRC 32681 & AB593740 & AB594807 \\
\hline Seimatosporium obtusum & CPC 12935 & JN871215 & JN871206 \\
\hline Seimatosporium parasiticum & NBRC 32682 & AB593741 & AB594808 \\
\hline Seimatosporium physocarpi & MFLUCC 14-0625 & KT198723 & KT198722 \\
\hline Seimatosporium pistaciae & CBS 138865 & КР004491 & KP004463 \\
\hline Seimatosporium rhombhisporum & MFLUCC 15-0543 & KR092780 & KR092792 \\
\hline Seimatosporium rosae & MFLUCC 14-0621 & KT198727 & KT198726 \\
\hline Seimatosporium vaccinii & ICMP 7003 & AF382374 & - \\
\hline Seimatosporium vitis & MFLUCC 14-0051 & KR920362 & KR920363 \\
\hline Seimatosporium walkeri & СРC 17644 & JN871216 & JN871207 \\
\hline Seimatosporium cornicola & MFLUCC 14-0448 & & KU974967 \\
\hline Seimatosporium quercina & MFLUCC 14-1198 & KU974964 & KU974965 \\
\hline
\end{tabular}


Independent Bayesian phylogenetic analyses were performed in MrBayes v. 3.1.2 (Huelsenbeck and Ronquist 2001) using a uniform [GTR+I+G] model. Posterior probabilities (PP) (Rannala and Yang 1996, Zhaxybayeva and Gogarten 2002) were determined by Markov Chain Monte Carlo sampling (BMCMC) in MrBayes v. 3.0b4 (Huelsenbeck and Ronquist 2001). Six simultaneous Markov chains were run for 10,000,000 generations and trees were sampled every 100th generation (resulting in 10,000 total trees). Phylogenetic trees were visualized with Treeview v. 1.6.6 (Page 1996). Bootstrap values of MP analyses (equal or above 70\%) and BYPP with those equal or greater than 0.95 are shown on the upper branches.

\section{Results}

\section{Phylogenetic analyses}

The combined LSU and ITS data set consists of 67 strains with Pseudopestalotiopsis theae (MFLUCC 12-0055) as the outgroup taxon. The data set consists of 1362 characters of which 1103 are constant, 83 are variable parsimony-uninformative characters and 176 are parsimonyinformative characters. One of the 16 equally most parsimonious trees is shown in Fig. 1.

Seimatosporium sensu stricto separates from Discosia sensu stricto with high bootstrap values and PP values (100\% and 1.00). A new strain, MFLUCC 14-0448 grouped with Seimatosporium pseudocornii (MFLUCC 13-0529) with high bootstrap values and PP values (86\% and 1.00).

\section{Taxonomy}

Seimatosporium pseudoglandigenum Wijayaw. \& E. Camporesi, sp. nov

Facesoffungi number: FoF 02076

Index Fungorum number: IF552047

Etymology: Named as it morphologically resembles Seimatosporium glandigenum

Holotype: MFLU 16-0837

Saprobic or endophytic on leaves of Quercus cerris L. Sexual morph: Undetermined. Asexual morph: Conidiomata 150-300 $\mu \mathrm{m}$ diam., 100-150 $\mu \mathrm{m}$ high, acervular, unilocular, subglobose to globose, superficial, gregarious, dark brown to black, apapillate ostiolate. Conidiomata wall multi-layered, outer wall thick, composed of brown cells of textura angularis, inner wall thin, hyaline, composed of hyaline cells of textura angularis. Paraphyses absent. Conidiophores 5-30 × 2-4 $\mu \mathrm{m}$, long, cylindrical, branched, hyaline, smooth-walled. Conidiogenous cells holoblastic, simple, integrated, determinate, hyaline. Conidia $15-23 \times 5-8 \mu \mathrm{m}$ $(\overline{\mathrm{x}}=19.14 \times 6.35 \mu \mathrm{m}, \mathrm{n}=20)$, obovoid to fusiform, or cymbiform, obtuse apex, straight to slightly curved, with 3 transverse septa, septa dark brown, constricted at the septa or continuous, eguttulate, medium brown to golden brown, with hyaline to sub-hyaline basal and apical cell, smooth-walled.

Material examined - Italy, Forlì-Cesena [FC] Province, near San Paolo in Alpe - Santa Sofia, on decaying leaves of Quercus cerris L. (Fagaceae), E. Camporesi, 15 December 2013, IT 1577, MFLU 16-0837, holotype.

Notes - We made several attempts to isolate this taxon in different media, but were unsuccessful. Hence, we compare our new collection with related species by host association (Sutton 1980, Nag Raj 1993, Farr and Rossman 2016). Taxa reported from Quercus species are summarized in Table 2. Moreover, we compared our collection with other Seimatosporium species with 3 transverse septa (Sutton 1980, Nag Raj 1993), but our new collection is morphologically distinct. 


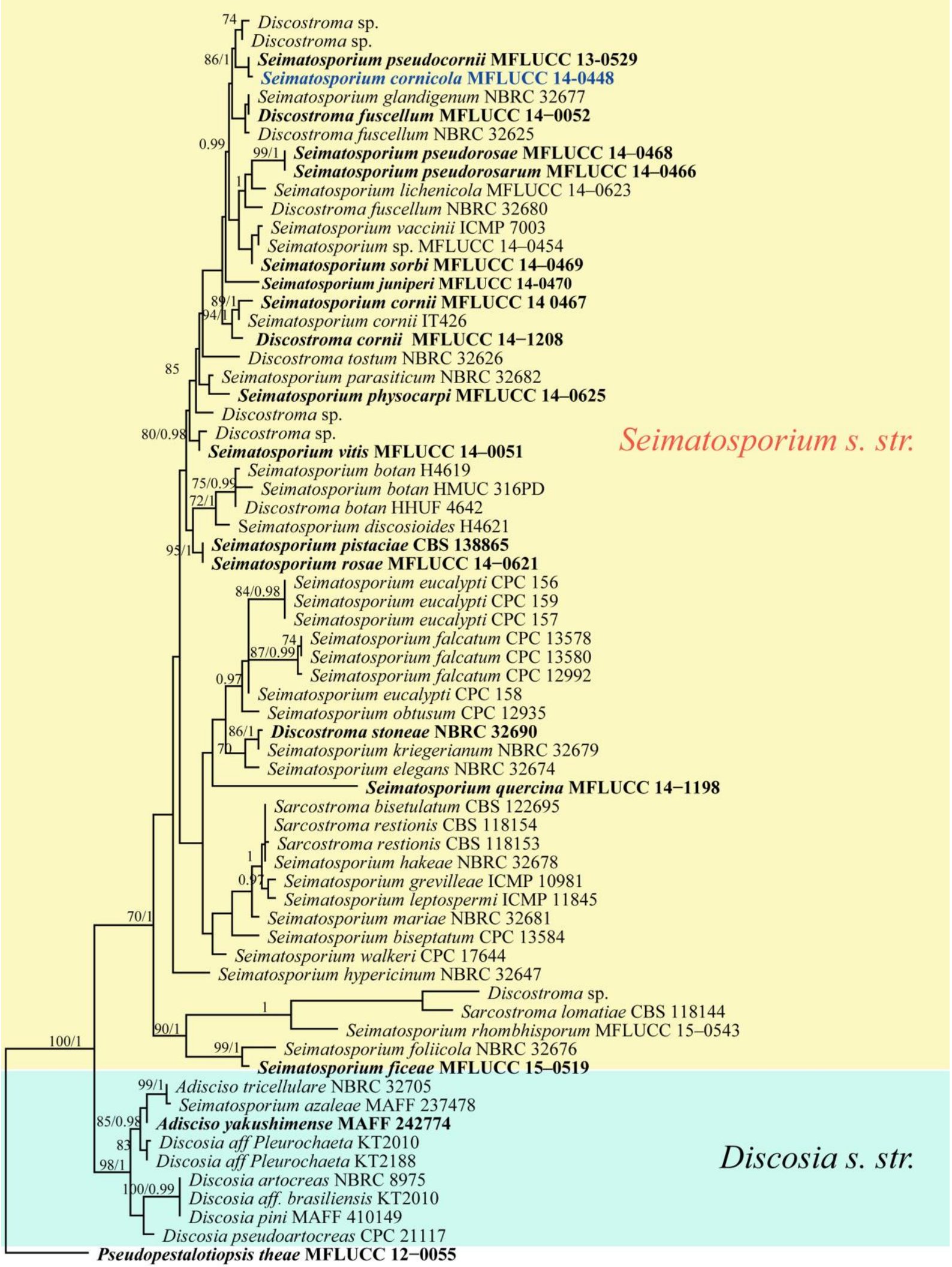

10

Fig. 1 - One of the 16 equally most parsimonious trees obtained from combined analyses set of ITS and LSU sequence data. $(\mathrm{CI}=0.557, \mathrm{RI}=0.776, \mathrm{RC}=0.443, \mathrm{HI}=0.443)$. MP values $(>70 \%)$ resulting from 1000 bootstrap replicates and Bayesian posterior probabilities above 0.95 are given at the nodes. The tree is rooted to Pseudopestalotiopsis theae (MFLUCC 12-0055). Ex-type strains are in bold and newly introduced species is in blue. 
Table 2 Seimatosporium species reported from Quercus spp.

\begin{tabular}{|c|c|c|c|c|}
\hline $\begin{array}{l}\text { Seimatosporium } \\
\text { spp. }\end{array}$ & Spore dimensions & Country & $\begin{array}{l}\text { Host }- \text { Quercus } \\
\text { spp. }\end{array}$ & Reference \\
\hline $\begin{array}{l}\text { S. caninum } \\
\text { (Brunaud) B. } \\
\text { Sutton }\end{array}$ & $\begin{array}{l}9.5-12 \mu \mathrm{m} \times 4.5-5.5 \\
\mu \mathrm{m}\end{array}$ & India & Q. incana & Sutton 1980 \\
\hline $\begin{array}{l}\text { S. glandigenum } \\
\text { (Bubák \& Gonz. } \\
\text { Frag.) B. Sutton }\end{array}$ & $15-18 \mu \mathrm{m} \times 5-6.5 \mu \mathrm{m}$ & Spain & Q. ballota & Sutton 1980 \\
\hline $\begin{array}{l}\text { S. lichenicola } \\
\text { (Corda) } \\
\text { Shoemaker \& E. } \\
\text { Müll. }\end{array}$ & $\begin{array}{l}13-15 \mu \mathrm{m} \times 5.5-6.5 \\
\mu \mathrm{m}\end{array}$ & Italy & Q. ilex & Sutton 1980 \\
\hline
\end{tabular}

Seimatosporium caninum has only 2-septate conidia (Sutton 1980), thus it is distinct from our collection, which has 3-septate conidia. Seimatosporium glandigenum $(15-18 \times 5-6.5 \mu \mathrm{m})$ has a similar conidial morphology with our taxon $(15-23 \times 5-8 \mu \mathrm{m})$. However, our collection has higher variation in both conidial width and length. Sutton (1980) did not mention the slightly curved conidia in $S$. glandigenum, but our new species has slightly curved conidia.

Seimatosporium cornicola Wijayaw. \& E. Camporesi, sp. nov

Facesoffungi number: FoF 02077

Index Fungorum number: IF552048

Etymology: Named after the host genus Cornus

Holotype: MFLU 16-0701

Saprobic on dead branches of Cornus sanguinea. Sexual morph: Undetermined. Asexual morph: Conidiomata 330-400 $\mu \mathrm{m}$ diam., 220-250 $\mu \mathrm{m}$ high, acervular, superficial, solitary to gregarious, black, apapillate ostiolate. Conidiomata wall multi-layered, outer wall thick, composed of brown cells of textura angularis, inner wall thin, hyaline. Conidiophores $25-55 \times 2-4 \mu \mathrm{m}$, long, cylindrical, branched, hyaline, smooth-walled. Conidiogenous cells holoblastic, simple, integrated, determinate, hyaline. Conidia 34-51 $\times 13-18 \mu \mathrm{m}(\overline{\mathrm{x}}=41.86 \times 16.1 \mu \mathrm{m}, \mathrm{n}=20)$, fusiform or obovoid, base truncate, straight, with 3 transverse septa, dark septa brown, constricted at septa, guttulate when immature, medium brown, with hyaline to subhyaline basal cell, smooth-walled, appendage absent.

Culture characteristics - On PDA slow growing, attaining a diam. of $2.5 \mathrm{~cm}$ in 7 days at 18 ${ }^{\circ} \mathrm{C}$, white to pale brown from above, greyish white from below, with sparse mycelium, flat, margin uneven.

Table 3 Seimatosporium spp. reported from Cornus spp.

\begin{tabular}{|c|c|c|c|}
\hline $\begin{array}{l}\text { Seimatosporium } \\
\text { spp. }\end{array}$ & Spore dimensions & Country & Reference \\
\hline S. lichenicola & $\begin{array}{l}13-15 \times 5.5-6.5 \\
\mu \mathrm{m} \text { fide Sutton } \\
1980\end{array}$ & Ukraine & $\begin{array}{l}\text { Farr and Rossman } \\
2016\end{array}$ \\
\hline $\begin{array}{l}\text { S. salicinum } \\
\text { (Corda) Nag Raj }\end{array}$ & $\begin{array}{l}11-17 \times 4-6 \mu \mathrm{m} \\
\text { fide Nag Raj } 1993\end{array}$ & Ukraine & $\begin{array}{l}\text { Farr and Rossman } \\
2016\end{array}$ \\
\hline $\begin{array}{l}\text { S. corni Wijayaw. } \\
\text { et al. }\end{array}$ & $21-29 \times 9-11 \mu \mathrm{m}$ & Italy & $\begin{array}{l}\text { Senanayake et al. } \\
2015\end{array}$ \\
\hline $\begin{array}{l}\text { S. pseudocornii } \\
\text { Wijayaw. et al. }\end{array}$ & $31-42 \times 5-7 \mu \mathrm{m}$ & Italy & $\begin{array}{l}\text { Zhao et al. in } \\
\text { prep. }\end{array}$ \\
\hline S. cornicola & $\begin{array}{l}34-51 \times 13-18 \\
\mu \mathrm{m}\end{array}$ & Italy & In this study \\
\hline
\end{tabular}




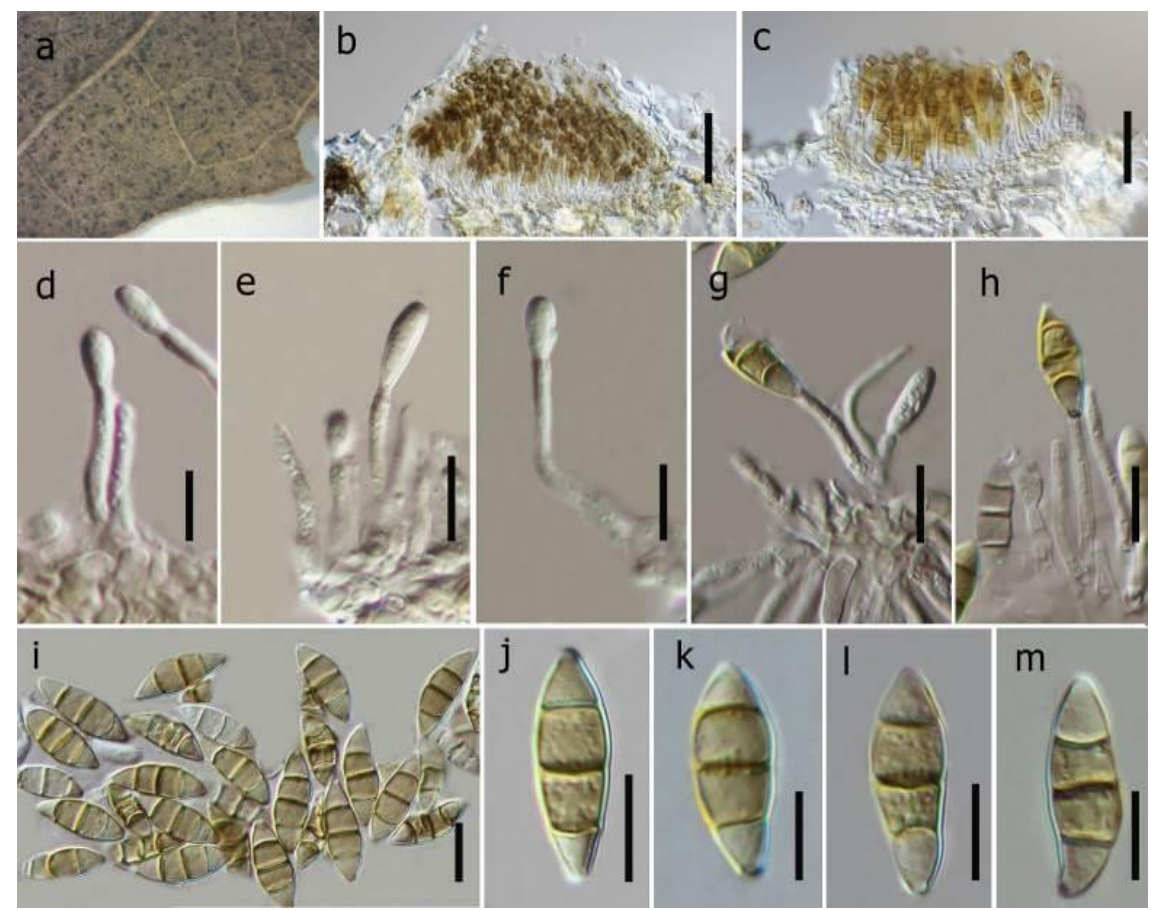

Fig. 2 - Seimatosporium pseudoglandigenum (holotype). a Conidiomata on leaves of Quercus cerris. b, c Vertical sections of conidiomata. d-h Different stages of conidiogenesis. i-m Conidia. Scale bars: $b, c=100 \mu \mathrm{m}, \mathrm{d}-\mathrm{m}=10 \mu \mathrm{m}$.

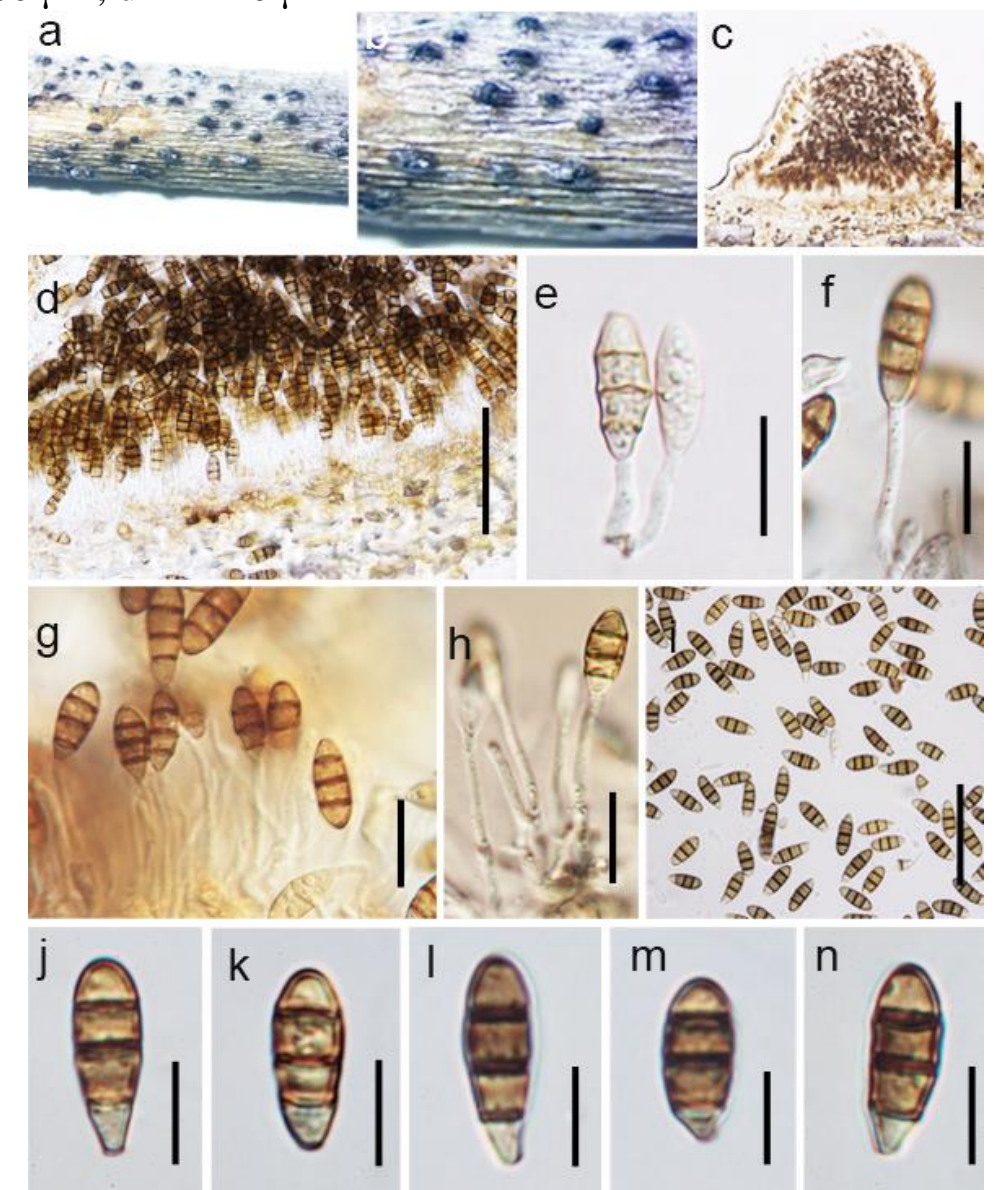

Fig. 3 - Seimatosporium cornicola (holotype). a, b Conidiomata on dead branch of Cornus sanguinea. c, d Vertical sections of conidiomata. e-h Developing conidia attach to conidiogenous. i-n Conidia. Scale bars: $\mathrm{c}=200 \mu \mathrm{m}, \mathrm{d}=150 \mu \mathrm{m}, \mathrm{e}-\mathrm{h}=30 \mu \mathrm{m}, \mathrm{i}=100 \mu \mathrm{m}, \mathrm{j}-\mathrm{n}=35 \mu \mathrm{m}$. 
Material examined - Italy, Forlì-Cesena [FC] Province, Camposonaldo - Santa Sofia, on dead branch of Cornus sanguinea L. (Cornaceae), E. Camporesi, 17 March 2012, IT 171 (MFLU 16-0701, holotype); ex-type living cultures MFLUCC 14-0448, GUCC IT 171.

Notes - Several Seimatosporium species have been recorded from Cornus spp. (Sutton 1980, Nag Raj 1993, Senanayake et al. 2015, Farr and Rossman 2016) (Table 3).

In phylogenetic analyses, the new collection clusters with Seimatosporium pseudocornii (MFLUCC 13-0529) with high bootstrap values and PP values (86\% and 1.00 respectively). However, in conidial morphology, both species are distinct (see Table 3). Seimatosporium pseudocornii has shorter conidiophores than in $S$. cornicola $(5-30 \mu \mathrm{m}$ vs. $25-55 \mu \mathrm{m})$. Hence, we introduce a new scientific name to accommodate our new collection.

\section{Discussion}

The genus Seimatosporium comprises 86 epithets in Index Fungorum (2016), but only a few species have sequence data. Norphanphoun et al. (2015) designated the epitype (MFLUCC 14-0621) of S. rosae, the type species of Seimatosporium and phylogenetic analyses confirmed Seimatosporium and Discostroma are a monophyletic clade. Senanayake et al. (2015) showed that Seimatosporium sensu stricto grouped with Discosia, thus they introduced Discosiaceae to accommodate them. Wijayawardene et al. (2016) also agreed with the findings of Senanayake et al. (2015). Wijayawardene et al. (2016) used a combined LSU, ITS, SSU, $\beta$-tubulin and RPB2 data set in their analyses and confirmed the familial arrangements of Senanayake et al. (2015) in Xylariomycetidae.

In this study, we introduce two Seimatosporium species based on morphology or morphophylogenetic analyses. Seimatosporium pseudoglandigenum lacks sequence data, thus we have compared it with other taxa in Sutton (1980), Ariyawansa et al. (2015), Senanayake et al. (2015) and Zhao et al. (in prep). Seimatosporium cornicola groups with Seimatosporium pseudocornii (MFLUCC 13-0529) with high bootstrap and PP support (86\% and 1.00 respectively). As they are distinct in morphology and show different branch lengths, we introduce $S$. cornicola as a new species.

Ariyawansa et al. (2015), Norphanphoun et al. (2015) and Goonasekara et al. (2016) used LSU and ITS sequence data in their analyses and are only available for most Seimatosporium species in GenBank (Crous et al. 2014, Ariyawansa et al. 2015, Norphanphoun et al. 2015, Goonasekara et al. 2016, Zhao et al. in prep.). However, it is much more reliable to include a protein gene as only LSU and ITS genes do not show high resolution among species. As an example, Seimatosporium pseudorosarum Wijayaw. et al. (Ariyawansa et al. 2015) groups with $S$. pseudorosae Wijayaw. et al. (Zhao et al. in prep.). Both species are morphologically distinct, as the former species only has basal appendages, while the latter has both apical and basal appendages. Since most of the species lack protein genes, we recommend relying both on morphology and molecular analyses, prior to introducing new species of Seimatosporium.

\section{Acknowledgements}

The research is supported by the projects of National Natural Science Foundation of China (No. 31560489), the Science and Technology Department of Guizhou Province [2012]4012 and Fundamental Research on Science and Technology, Ministry of Science and Technology of China (2014FY120100).

\section{References}

Alves A, Correia A, Luque J, Phillips AJL. 2004 - Botryosphaeria corticola, sp. nov. on Quercus species, with notes and description of Botryosphaeria stevensii and its anamorph, Diplodia mutila. Mycologia 96, 598-613.

Ariyawansa HA, Hyde KD, Jayasiri SC, Buyck B, Chethana KWT, Dai DQ, Dai YC, Daranagama DA, Jayawardena RS, Lücking R, Ghobad-Nejhad M, Niskanen T, Thambugala KM, Voigt 
K, Zhao RL, Li GJ, Doilom M, Boonmee S, Yang ZL, Cai Q, Cui YY, Bahkali AH, Chen J, Cui BK, Chen YY, Monika CD, Dissanayake AJ, Ekanayaka AH, Hashimoto A, Hongsanan S, Jones EBG, Larsson E, Li WJ, Li QR, Liu JK, Luo ZL, Maharachchikumbura SSN, Mapook A, McKenzie EHC, Norphanphoun C, Konta S, Pang KL, Perera RH, Phookamsak R, Phukhamsakda C, Pinruan U, Randrianjohany E, Singtripop C, Tanaka K, Tian CM, Tibpromma S, Abdel-Wahab MA, Wanasinghe DN, Wijayawardene NN, Zhang JF, Zhang H, Abdel-Aziz FA, Wedin M, Westberg M, Ammirati JF, Bulgakov TS, Lima DX, Callaghan TM, Callac P, Chang CH, Coca LF, Dal-Forno M, Dollhofer V, Fliegerová K, Greiner K, Griffith GW, Ho HM, Hofstetter V, Jeewon R, Kang JC, Wen TC, Kirk PM, Kytövuori I, Lawrey JD, Xing J, Li H, Liu ZY, Liu XZ, Liimatainen K, Lumbsch HT, Matsumura M, Moncada B, Moncada S, Parnmen S, de Azevedo Santiago ALCM, Sommai S, Song Y, de Souza CAF, de Souza-Motta CM, Su HY, Suetrong S, Wang Y, Wei SF, Yuan HS, Zhou LW, Réblová M, Fournier J, Camporesi E, Luangsa-ard JJ, Tasanathai K, Khonsanit A, Thanakitpipattana D, Somrithipol S, Diederich P, Millanes AM, Common RS, Stadler M, Yan JY, Li XH, Lee HW, Nguyen TTT, Lee HB, Battistin E, Marsico O, Vizzini A, Vila J, Ercole E, Eberhardt U, Simonini G, Wen HA, Chen XH. 2015 - Fungal diversity notes 111-252 - taxonomic and phylogenetic contributions to fungal taxa. Fungal Diversity $75,1-248$.

Barber PA, Crous PW, Groenewald JZ, Pascoe IG, Keane P. 2011 - Reassessing Vermisporium (Amphisphaeriaceae), a genus of foliar pathogens of Eucalypts. Persoonia 27, 90-118. DOI: 10.3767/003158511X617381.

Chomnunti P, Hongsanan S, Hudson BA, Tian Q, Peršoh D, Dhami MK, Alias AS, Xu J, Liu X, Stadler M, Hyde KD. 2014 - The Sooty Moulds. Fungal Diversity 66, 1-36.

Corda ACJ. 1833 - Deutschlands Flora, Abt. III. Die Pilze Deutschlands 3-13, 65-96.

Farr DF, Rossman AY. 2016 - Fungal Databases, Systematic Mycology and Microbiology Laboratory, ARS, USDA. Retrieved January 11, 2015, from http://nt.ars-grin.gov/fungaldatabases/

Goonasekara ID, Maharachchikumbura SSN, Wijayawardene NN, Phookamsak R, Schumacher RK, Bahkali AH, Hyde KD. 2016 - Seimatosporium quercina sp. nov. (Discosiaceae) on Quercus robur from Germany. Phytotaxa 255(3), 240-248.

Huelsenbeck JP, Ronquist F. 2001 - MRBAYES: Bayesian inference of phylogenetic trees. Bioinformatics 17, 754-755.

Index Fungorum 2016. http://www.indexfungorum.org/Names/Names.asp

Jayasiri SC, Hyde KD, Ariyawansa HA, Bhat DJ, Buyck B, Cai L, Dai YC, Abd-Elsalam KA, Ertz D, Hidayat I, Jeewon R, Jones EBG, Bahkali AH, Karunarathna SC, Liu JK, Luangsa-ard JJ, Lumbsch HT, Maharachchikumbura SSN, McKenzie EHC, Moncalvo JM, Ghobad-Nejhad M, Nilsson H, Pang KL, Pereira OL, Phillips AJL, Raspé O, Rollins AW, Romero AI, Etayo J, Selçuk F, Stephenson SL, Suetrong S, Taylor JE, Tsui CKM, Vizzini A, AbdelWahab MA, Wen TC, Boonmee S, Dai DQ, Daranagama DA, Dissanayake AJ, Ekanayaka AH, Fryar SC, Hongsanan S, Jayawardena RS, Li WJ, Perera RH, Phookamsak R, de Silva NI, Thambugala KM, Tian Q, Wijayawardene NN, Zhao RL, Zhao Q, Kang JC, Promputtha I. 2015 - The Faces of Fungi database: fungal names linked with morphology, phylogeny and human impacts. Fungal Diversity 74, 3-18.

Katoh K, Misawa K, Kuma K, Miyata T. 2002 - MAFFT: a novel method for rapid multiple sequence alignment based on fast Fourier transform. Nucleic Acids Research 30, 30593066.

Katoh K, Toh H. 2008 - Recent developments in the MAFFT multiple sequence alignment program. Briefings in Bioinformatics 9, 276-285.

Nag Raj TR. 1993 - Coelomycetous anamorphs with appendage-bearing conidia. Mycologue Publications, Waterloo, Ontario, Canada. 
Norphanphoun C, Maharachchikumbura SSN, Daranagama A, Bulgakov TS, Bhat DJ, Bahkali AH, Hyde KD. 2015 - Towards a backbone tree for Seimatosporium, with S. physocarpi sp. nov. Mycosphere 6(3), 385-400.

Page RDM. 1996 - Tree View: an application to display phylogenetic trees on personal computers. Computer Applications in the Biosciences 12, 357-358.

Senanayake IC, Maharachchikumbura SSN, Hyde KD, Bhat JD, Jones EBG, McKenzie EHC, Dai DQ, Daranagama DA, Dayarathne M C, Goonasekara ID, Konta S, Li WJ, Shang QJ, Stadler M, Wijayawardene NN, Xiao YP, Norphanphoun C, Li Q, Liu XZ, Bahkali AH, Kang JC, Wang Y, Wen TC, Wendt L, Xu JC, Camporesi E. 2015 - Towards unraveling relationships in Xylariomycetidae (Sordariomycetes). Fungal Diversity 73, 73-144. DOI:10.1007/s13225-015-0340-y.

Sutton BC. 1980 - The Coelomycetes. Fungi imperfecti with pycnidia, acervuli and stromata. Commonwealth Mycological Institute, Kew.

Swofford DL. 2002 - PAUP: phylogenetic analysis using parsimony, version 4.0 b10. Sinauer Associates, Sunderland.

Tanaka K, Endo M, Hirayama K, Okane I, Hosoya T, Sato T. 2011 - Phylogeny of Discosia and Seimatosporium, and introduction of Adisciso and Immersidiscosia genera nova. Persoonia 26, 85-98.

Vilgalys R, Hester M. 1990 - Rapid genetic identification and mapping of enzymatically amplified ribosomal DNA from several Cryptococcus species. Journal of Bacteriology 172, 4238 4246.

White TJ, Bruns T, Lee J, Taylor SB. 1990 - Amplification and direct sequencing of fungal ribosomal RNA genes for phylogenetics. In: Innis MA, Gelfand DH, Sninsky JJ, White TJ (eds), PCR protocols: a guide to methods and applications, 315-322. Academic Press, San Diego, California, USA.

Wijayawardene NN, Hyde KD, Wanasinghe DN, Papizadeh M, Goonasekara ID, Camporesi E, Bhat DJ, McKenzie EHC, Phillips AJL, Diederich P, Tanaka K, Li WJ, Tangthirasunun N, Phookamsak R, Dai DQ, Dissanayake AJ, Weerakoon G, Maharachchikumbura SSN, Hashimoto A, Matsumura M, Bahkali AH, Wang Y. 2016 - Taxonomy and phylogeny of dematiaceous coelomycetes. Fungal Diversity (in press). DOI 10.1007/s13225-016-0360-2 